Génét. Sél. Evol., 1987, 19 (3), 351-364

\title{
Héritabilité réalisée pour la taille de portée dans la sélection de truies dites "hyperprolifiques"
}

\author{
Pascale LE ROY*, C. LEGAULT*, J. GRUAND** et L. OLLIVIER* \\ *I.N.R.A., Centre de Recherches de Jouy-en-Josas, Station de Génétique quantitative et appliquée, \\ F78350 Jouy-en-Josas \\ ** Station expérimentale de Sélection porcine, F86480 Rouillé
}

\begin{abstract}
Résumé
Une expérience de sélection sur la prolificité est menée depuis 1973 au Centre d'Insémination Artificielle porcine de l'I.N.R.A., à Rouillé (Vienne). Une lignée de verrats Large White (appelés H) est créée par sélection de truies de prolificité extrême (dites "hyperprolifiques ») dans une grande population et accouplement de leur fils à d'autres truies hyperprolifiques de la même population. Par ce système de croisement en retour, répété chaque année, le niveau génétique espéré des verrats $\mathbf{H}$ atteint progressivement celui des femelles hyperprolifiques. L'efficacité de ce plan de sélection est évaluée par comparaison, dans des élevages de production, de filles de verrats H à des truies d'une autre lignée Large White, utilisée comme témoin (T). Au total, les portées de 850 truies, issues de 24 verrats H sélectionnés de 1973 à 1983 et de 118 verrats T contemporains, sont analysées pour les variables "nés totaux " (NT) et "nés vivants » (NV). Dans une première analyse, la taille de portée est considérée comme une variable unique, dont la répétabilité est supposée égale à 0,15 . En prenant comme variable indépendante le critère de sélection $\mathrm{D}$ des truies hyperprolifiques $(\mathrm{D}=\mathrm{n} \overline{\mathrm{d}} /(1+0,15(\mathrm{n}-1))$, où $\overline{\mathrm{d}}$ est la supériorité moyenne de NT sur $\mathrm{n}$ portées), l'héritabilité réalisée $h_{r}{ }^{2}$ est estimée par régression en fonction de la différentielle de sélection cumulée des verrats $H$. Les valeurs de $h_{r}{ }^{2}(0,14 \pm 0,05$ et $0,10 \pm 0,05$ respectivement pour NT et NV) situent l'efficacité globale du schéma de sélection, i.e. un gain attendu de 0,8 porcelet en $N T$ chez les filles de verrats $H$ pour $D=12$. L'analyse révèle cependant une hétérogénité significative de $h_{r}{ }^{2}$ selon le rang de portée $(0,03,0,26$ et 0,18 pour NT, respectivement pour les rangs 1,2 et 3 et plus). Par ailleurs, les héritabilités estimées par régression mère-fille $(0,02 \pm 0,03$ pour NT et $0,05 \pm 0,03$ pour NV) sont inférieures à $h_{r}^{2}$, ce qui suggère l'existence d'effets maternels défavorables. Une seconde analyse est réalisée pour tenter d'estimer les paramètres génétiques réalisés en considérant les portées successives d'une truie comme des caractères différents. Bien qu'estimés avec une faible précision, les paramètres obtenus indiquent que la taille de la $1^{\text {re }}$ portée est moins héritable que les autres, et que des corrélations génétiques sensiblement inférieures à 1 peuvent exister entre les portées successives. Les causes possibles de ces différences et les conséquences à en attendre pour la sélection sont discutées.
\end{abstract}

Mots clés: Héritabilité réalisée, taille de portée, porc, effet maternel.

\section{Summary}

Realized heritability for litter size in selection of " hyperprolific " sows

A selection experiment for prolificacy is carried on since 1973 at the pig artificial insemination centre of Rouillé (Vienne, France). A Large White boar line (called $\mathbf{H}$ ) is created by selecting extremely prolific sows (so-called "hyperprolific») in a large population and mating their sons to 
other hyperprolific sows from the same population. By repeating every year this type of backcross, the genetic merit for prolificacy of the $\mathrm{H}$ boars is expected to progressively reach the hyperprolific level. The efficiency of such a selection scheme is assessed by comparing daughters of $\mathrm{H}$ boars to daughters of another Large White boar line, used as a control (C), under commercial farm conditions. Altogether, litters by 850 sows, from $24 \mathrm{H}$ boars selected over the period 1973-1983 and from 118 contemporary C boars, are analysed for total born (NT) and born alive (NT). In a first analysis, litter size is considered as a single trait, with repeatability 0.15 . Using the selection criterion $D$ of hyperprolific sows $(D=n \bar{d} /(1+0.15(n-1))$, where $\bar{d}$ is the superiority in NT averaged over $n$ litters) as the independent variable, realized heritability $h_{\mathrm{r}}{ }^{2}$ is estimated by regression on the cumulated selection differential of $\mathrm{H}$ boars. The values of $\mathrm{h}_{\mathrm{r}}{ }^{2}(0.14 \pm 0.05$ and $0.10 \pm 0.05$ for NT and NV respectively) measure the global efficiency of the selection scheme, i.e. an estimated gain of 0.8 piglet in $N T$ on daughters of $H$ boars assuming $D=12$. However, the analysis also shows a significant heterogeneity of $\mathrm{h}_{\mathrm{r}}{ }^{2}$ according to parity $(0.03,0.26$ and 0.18 for NT in parity 1,2 and 3 or more, respectively). Furthermore, heritabilities estimated by daughter-dam regression $\left(0.02 \pm 0.03\right.$ and $0.05 \pm 0.03$ for NT and NV respectively) are lower than $h_{r}^{2}$, which suggests the existence of unfavourable maternal effects. An attempt is made, in a second analysis, to estimate realized genetic parameters by considering successive litters as different traits. Though rather poorly estimated, these parameters suggest a lower heritability for first litters as compared to the others, and possible low genetic correlations between successive litters. The possible causes of those differences and their consequences for selection are discussed.

Key words : Realized heritability, litter size, pig, maternal effect.

\section{Introduction}

L'accroissement de la productivité numérique des truies observé en France durant ces 15 dernières années, qui correspond à un gain annuel évalué à 0,3 porcelet sevré par truie et par an, est attribué essentiellement à une accélération du rythme de reproduction (réduction de la durée d'allaitement et de l'intervalle sevrage-fécondation) et à une meilleure survie des porcelets de la naissance au sevrage, la taille de portée à la naissance étant demeurée pratiquement constante au cours de cette période (Noguera et al., 1983 ; Ollivier et al., 1986). L'amélioration génétique de ce dernier critère est donc considérée actuellement comme un objectif prioritaire pour l'obtention de nouveaux gains de productivité.

Malgré la faible héritabilité de la prolificité $\left(\mathrm{h}^{2}\right.$ voisin de 0,10$)$, un progrès génétique substantiel peut théoriquement être obtenu par sélection, mais sa réalisation dans des troupeaux fermés de taille limitée se heurte à la difficulté d'appliquer une intensité de sélection suffisante (Ollivier \& BOLET, 1981). Le développement des programmes informatisés de gestion des troupeaux, en facilitant le criblage de grandes populations, permet de contourner cette difficulté et d'exploiter au mieux la variabilité phénotypique naturelle de la taille de la portée. C'est la base du système proposé par LEgAulT \& GRUAND (1976) pour détecter et exploiter génétiquement l'existence de truies dites « hyperprolifiques », qui correspondent à un taux de sélection sur la prolificité de l'ordre de 1 p. 1000. Les aspects théoriques de la méthode ont également été discutés récemment par BICHARD \& DAVID (1985). Une première analyse des réponses à ce type de sélection, dans les conditions d'un troupeau expérimental, a été présentée par LEGAULT et al. (1981) et BolET \& LEGAult (1982). L'objet du présent article est d'analyser les réponses obtenues dans les élevages de production, et de les confronter aux prédictions théoriques, basées sur les paramètres génétiques de la prolificité, suivant une méthodologie analogue à celle utilisée dans les expériences de sélection pour estimer les paramètres génétiques réalisés. 


\section{Matériel et méthodes}

\section{A. Protocole expérimental}

Le principe de l'expérience, tel qu'il a été exposé par LEGAULT \& GRUAND (1976), consiste à sélectionner dans une population des femelles de prolificité exceptionnelle, dites hyperprolifiques, dont les fils sont eux-mêmes accouplés à des truies hyperprolifiques de la même population pour engendrer la génération suivante de verrats. Ainsi, par croisements en retour successifs sur des femelles d'un niveau de prolificité donné, le niveau génétique des verrats atteint asymptotiquement celui de ces femelles. Ce schéma est appliqué en race Large White depuis 1973, à raison d'une génération de verrats par an, dans une expérience réalisée par les stations expérimentales I.N.R.A. de sélection porcine et d'insémination artificielle de Rouillé (Vienne).

La sélection des verrats de la lignée hyperprolifique (appelés $H$ ) est basée sur un critère de prolificité maternelle $\mathrm{D}$ défini comme suit :

$$
\mathrm{D}=\mathrm{n} \overline{\mathrm{d}} /[1+0,15(\mathrm{n}-1)]
$$

Ce critère prend en compte le nombre $\mathrm{n}$ de portées connues, un coefficient de répétabilité de 0,15 pour la taille de portée, et la supériorité phénotypique moyenne $\bar{d}$ (en nombre total de porcelets nés) des $\mathrm{n}$ portées connues relativement à une moyenne contemporaine. Celle-ci est la prolificité des mères d'un échantillon de verrats Large White (appelés T) contrôlés à la Station de Rouillé dans le cadre d'une expérience de sélection sur la vitesse de croissance du tissu maigre (Ollivier, 1985). Préalablement au calcul de $d$, des facteurs de correction de $+1,4$ et $+0,4$ sont appliqués respectivement aux $1^{\text {res }}$ et $2^{\text {es }}$ portées de chaque truie.

Les jeunes verrats des 2 lignées ( $\mathrm{H}$ et $\mathrm{T}$ ) sont sélectionnés à l'issue d'un contrôle individuel de croissance et d'adiposité, entre 30 et $85 \mathrm{~kg}$ de poids vif, et utilisés en insémination artificielle sur des truies Large White de la région. Un échantillon de leurs filles est soumis chaque année au même contrôle que celui indiqué ci-dessus pour les verrats et les femelles sélectionnées à l'issue de ce contrôle individuel sont inséminées et cédées, dès confirmation de la gestation, à des éleveurs de la région. Les portées produites dans ces élevages par les 2 types de femelles sont enregistrées de manière à évaluer les réponses à la sélection des verrats $H$.

L'analyse qui va être présentée concerne un effectif de 850 truies, ayant produit un total de 2323 portées entre 1975 et 1985 , et dont la distribution en fonction des origines paternelles et maternelles est indiquée au tableau 1. Les variables analysées sont le nombre total de porcelets nés (NT) et le nombre de porcelets nés vivants (NV) par portée.

\section{B. Méthodes d'analyse}

Les tailles des portées successives d'une femelle peuvent être considérées soit comme des expressions répétées d'un même caractère, soit comme un ensemble de caractères distincts. Dans l'analyse nous adopterons successivement ces 2 hypothèses, ce qui conduit donc à 2 approches différentes, une approche univariate et une approche multivariate. 
TABLEAU 1

Répartition des truies et des portées en fonction des origines paternelles et maternelles. Distribution of sows and litters according to paternal and maternal origins.

\begin{tabular}{|c|c|c|c|}
\hline Mère Père & $\begin{array}{c}\mathrm{T} \\
(\mathrm{N}=118)\end{array}$ & $\begin{array}{c}\mathrm{H} \\
(\mathrm{N}=24)\end{array}$ & $\begin{array}{c}\text { Total } \\
(N=142)\end{array}$ \\
\hline $\begin{array}{l}\mathrm{D}<8 \\
(\mathrm{~N}=483)\end{array}$ & $\begin{array}{c}682 \text { truies } \\
\text { avec } 1895 \text { portées }\end{array}$ & $\begin{array}{l}92 \text { truies } \\
\text { avec } 250 \text { portées }\end{array}$ & $\begin{array}{c}774 \text { truies } \\
\text { avec } 2145 \text { portées }\end{array}$ \\
\hline $\begin{array}{l}\mathrm{D}>8 \\
(\mathrm{~N}=39)\end{array}$. & $\begin{array}{l}46 \text { truies } \\
\text { avec } 109 \text { portées }\end{array}$ & $\begin{array}{l}30 \text { truies } \\
\text { avec } 69 \text { portées }\end{array}$ & $\begin{array}{c}76 \text { truies } \\
\text { avec } 178 \text { portées }\end{array}$ \\
\hline $\begin{array}{l}\text { Total } \ldots \\
(\mathrm{N}=522)\end{array}$ & $\begin{array}{c}728 \text { truies } \\
\text { avec } 2004 \text { portées }\end{array}$ & $\begin{array}{l}122 \text { truies } \\
\text { avec } 319 \text { portées }\end{array}$ & $\begin{array}{c}850 \text { truies } \\
\text { avec } 2323 \text { portées }\end{array}$ \\
\hline
\end{tabular}

$\mathrm{T}$ : lignée témoin, control line.

$\mathrm{H}$ : lignée hyperprolifique, hyperprolific line.

$\mathrm{D}$ : critère de sélection des mères, selection criterion of dams : formule (I).

\section{Approche univariate}

Dans le cas d'un caractère unique, de répétabilité donnée, Foulley (1981) a montré que la régression parent-descendant peut être estimée, lorsque le nombre $\mathrm{n}$ de répétitions varie chez le parent, en utilisant la variable $D$ définie en (1) comme variable indépendante. Cette méthode est retenue ici pour estimer la régression mère-fille, $\beta^{(\mathrm{d})}$, en attribuant à chaque mère la valeur $D$ correspondante. Pour les pères de la lignée $H$, il faut tenir compte de la sélection effectuée à chaque génération sur les mères des verrats $\mathrm{H}$, et de celle effectuée à la génération précédente dans le choix des pères. Chaque verrat est ainsi caractérisé par une différentielle de sélection $\mathrm{S}$, cumulée au cours des générations, qui est la moyenne de la différentielle $\mathrm{D}$ de sa mère et de celle de son père. La supériorité génétique attendue $R_{t}$ d'un verrat de l'année $t$, en fonction des sélections $D$ effectuées sur les truies aux années $t-1, t-2, \ldots 0$, et de l'héritabilité $\mathrm{h}^{2}$ du caractère, s'écrit alors :

$$
R_{t}=S_{t} h^{2}
$$

avec :

$$
S_{t}=\sum_{i=1}^{t}(1 / 2)^{i} D_{t-i}
$$

Par analogie, nous attribuons aux verrats $\mathrm{T}$ une valeur de $\mathrm{S}$ égale à la moitié du niveau de prolificité $\mathrm{D}$ de leur mère.

L'expression (2a) montre que, connaissant $R$, l'héritabilité réalisée $h_{r}{ }^{2}$ est obtenue par la régression $\beta^{(s)}$ de $R$ en fonction de $S$. Dans notre cas, puisque les verrats sont évalués sur la prolificité de leurs filles, $h_{\mathrm{r}}{ }^{2}=2 \beta^{(\mathrm{s})}$. suivant :

Les régressions $\beta^{(d)}$ et $\beta^{(s)}$ sont estimées simultanément selon le modèle linéaire 


$$
Y_{i j k}=\mu+p_{i}+n_{j}+\beta_{j}^{(s)} S_{i j k}+\beta_{j}^{(d)} D_{i j k}+e_{i j k}
$$

où $\quad \mu$ est l'espérance de la variable dépendante $Y_{\mathrm{ijk}}$ (NT ou NV),

$\mathrm{p}_{\mathrm{i}} \quad$ l'effet de la période semestrielle de mise bas (mai à octobre, novembre à avril) pour chacune des 11 années considérées (22 niveaux),

$\mathrm{n}_{\mathrm{j}} \quad$ l'effet $\mathrm{du}$ numéro de portée qui comprend 3 niveaux $\left(1^{\mathrm{re}}, 2^{\mathrm{e}}, 3^{\mathrm{e}}\right.$ et d'ordre supérieur)

et $\mathrm{e}_{\mathrm{ijk}}$ la résiduelle.

Ce modèle prend en compte la possibilité que les $\beta$ puissent varier selon le numéro de portée $\mathrm{j}$, et il permet donc de tester l'homogénéité de ces régressions.

\section{Approche multivariate}

La méthode précédente peut facilement se généraliser à la situation où les $\mathrm{n}$ portées sont considérées comme des caractères différents. La sélection effectuée sur les mères des verrats de la lignée $\mathrm{H}$ peut être ramenée à une sélection sur un indice à $\mathrm{n}$ caractères, dont les coefficients, représentés par le vecteur $\mathbf{D}$, sont obtenus à partir des $\mathrm{n}$ différentielles de sélection (d) et de la matrice des variances-covariances des $\mathbf{n}$ portées considérées (P), par la relation :

$$
\mathbf{D}=\mathbf{P}^{-1} \mathbf{d}
$$

La supériorité génétique $R_{t}$ d'un verrat pour une portée de rang quelconque s'exprime alors en fonction de $\mathbf{D}$ et du vecteur $\mathbf{C}$ des $\mathrm{n}$ covariances génétiques entre la portée considérée d'une part et les portées de rang 1 à n d'autre part, selon la formule suivante, qui a été démontrée dans un cas plus général par Ollivier (1985) :

$$
\mathrm{R}_{\mathrm{t}}=\mathrm{S}_{\mathrm{t}} \mathrm{C}
$$

avec :

$$
\mathbf{S}_{\mathrm{t}}=\sum_{\mathrm{i}=1}^{\mathrm{t}}(1 / 2)^{\mathrm{i}} \mathbf{D}_{\mathrm{t}-\mathrm{i}}
$$

De même que précédemment, le vecteur $\mathbf{S}$ attribué aux verrats $\mathrm{T}$ est égal à la moitié du vecteur $\mathbf{D}$ caractérisant leur mère.

L'expression (4a) montre que les régressions partielles $\boldsymbol{\beta}^{(\mathrm{s})}$ de $\mathrm{R}$ en fonction du vecteur $\mathbf{S}$ permettent d'estimer le vecteur $\mathbf{C}_{\mathrm{r}}$ des covariances génétiques réalisées. Comme précédemment dans le cas d'une variable, on a ici :

$$
\mathbf{C}_{\mathrm{r}}=2 \boldsymbol{\beta}^{(\mathrm{s})}
$$

Les vecteurs $\boldsymbol{\beta}^{(\mathrm{s})}$ et $\boldsymbol{\beta}^{(\mathrm{d})}$ peuvent être estimés simultanément selon un modèle analogue à (3), soit, pour une portée de rang $\mathrm{j}$ :

$$
\mathrm{Y}_{\mathrm{ik}}=\mu+\mathrm{p}_{\mathrm{i}}+\sum_{1=1}^{\mathrm{n}}\left[\beta_{1}^{(\mathrm{s})} \mathrm{S}_{\mathrm{ik}}^{(1)}+\beta_{1}^{(\mathrm{d})} \mathrm{D}_{\mathrm{ik}}^{(1)}\right]+\mathrm{e}_{\mathrm{ik}}
$$

où les $\mathbf{S}^{(1)}$ et les $\mathrm{D}^{(1)}$ sont les éléments respectifs des vecteurs $\mathbf{S}$ et $\mathbf{D}$ définis plus haut. Des valeurs nulles sont données aux différentielles d correspondant aux portées non prises en compte dans la sélection. 
3. Variance d'échantillonnage des coefficients de régression

Les modèles (3) et (5) supposent que les aléatoires résiduelles $\left(\mathrm{e}_{\mathrm{ijk}}\right.$ et $\mathrm{e}_{\mathrm{ik}}$ ) sont indépendantes entre elles et ne prennent donc pas en compte les corrélations qui existent entre demi-sœurs de père et entre portées successives d'une même fille, ce qui entraîne une sous-estimation des variances d'échantillonnage. Pour tenir compte de ce biais, les variances d'échantillonnage des $\beta^{(s)}$ sont multipliées par les coefficients suivants, qui dérivent de la formule générale donnée par OLLIVIER (1974),

pour le modèle (3) :

$$
1+\left[\sum_{i}\left(S_{i}-\bar{S}\right)^{2}\left(\rho^{\prime} \sum_{j} n_{i j}\left(n_{i j}-1\right)+\rho\left(n_{i o}^{2}-\sum_{j} n_{i j}^{2}\right)\right) / \sum_{i} n_{i o}\left(S_{i}-\bar{S}\right)^{2}\right]
$$

pour le modèle (5) :

$$
1+\rho\left[\sum_{i}\left(S_{i}-\bar{S}\right)^{2}\left(n_{i}^{2}-1\right) / \sum_{i} n_{i}\left(S_{i}-\bar{S}\right)^{2}\right]
$$

où :

$\rho$ est la corrélation entre les aléatoires résiduelles de 2 filles du père $\mathrm{i}$ : $\rho=\left(0,25 h^{2}-\beta^{2}\right) /\left(1-\beta^{2}\right)$,

$\rho^{\prime}$ la corrélation entre les aléatoires résiduelles de 2 portées de la même fille : $\rho^{\prime}=\left(0,15-\beta^{2}\right) /\left(1-\beta^{2}\right)$,

$n_{i j}$ le nombre de portées de la fille j du père $i\left(n_{i o}=\sum_{j} n_{i j}\right)$, et avec $\bar{S}=\sum_{i} n_{i o} S_{i} / \sum_{i} n_{i o}$.

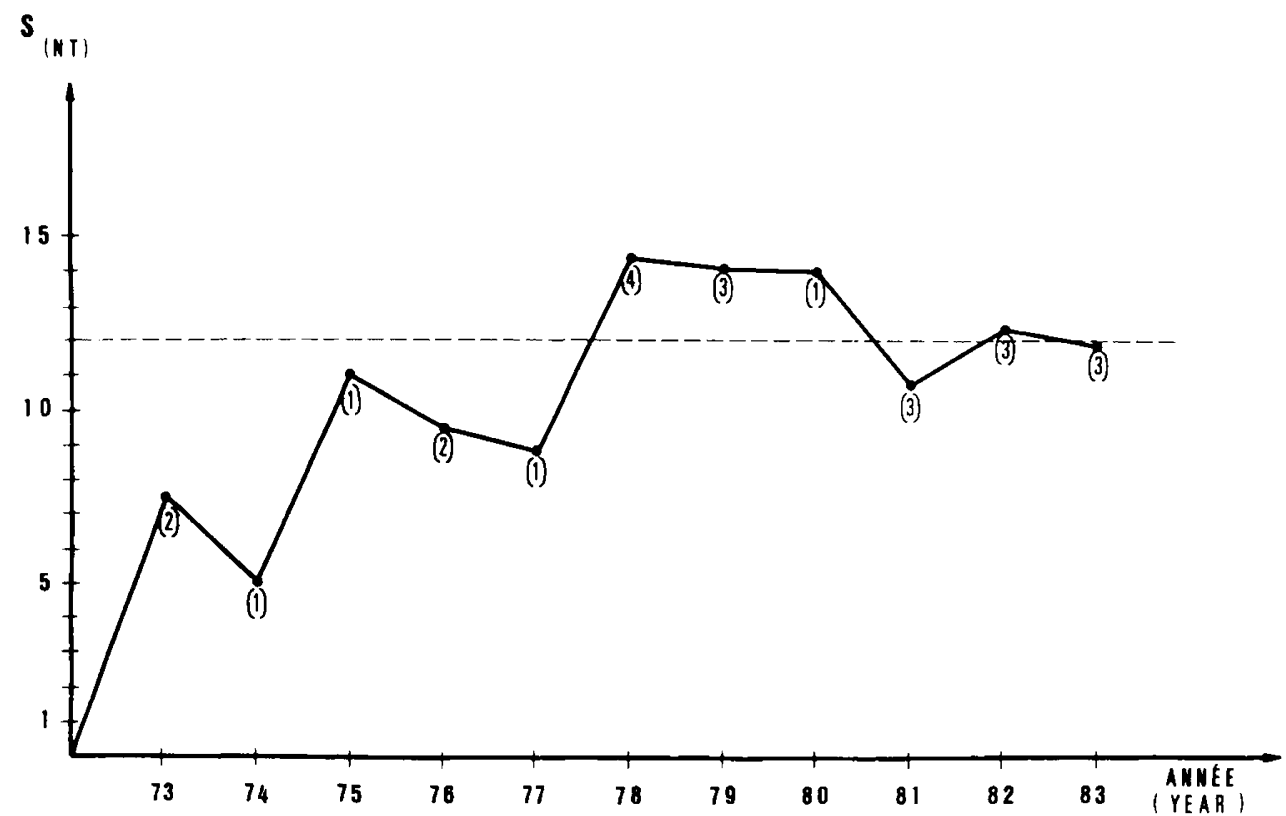

FIG. 1

Moyenne des différentielles de sélection cumulées (S) des verrats $H$ selon l'année (entre parenthèses: nombre de verrats).

Mean of cumulated selection differentials ( $S$ ) of $H$ boars by year (between brackets : number of boars). 


\section{Résultats}

\section{A. Approche univariate}

L'évolution de 1973 à 1983 de la moyenne des différentielles de sélection des verrats de la lignée $H$, calculées suivant l'expression (2b), est donnée dans la figure 1 . Elle montre qu'à partir de 1975 on peut considérer que «l'asymptote » fixée comme objectif (correspondant à $\mathrm{D}=12$ ) est atteinte, avec cependant des fluctuations notables selon l'année.

Les résultats de l'analyse de variance suivant le modèle univariate (3) sont présentés au tableau 2. En ce qui concerne la variable NT, l'effet du père est très hautement significatif en moyenne mais l'analyse révèle une hétérogénéité significative des coefficients de régression selon le numéro de portée. Par contre, les régressions mère-fille n'atteignent pas le seuil de signification et elles ne diffèrent pas significativement selon le numéro de portée. Les résultats de la variable NV sont en accord avec ceux de NT, à l'exception du test d'hétérogénéité de l'effet du père selon le numéro de portée, qui n'est pas significatif, et de l'effet de la période semestrielle qui est plus réduit que pour NT et devient de ce fait non significatif.

Les héritabilités déduites des coefficients de régression estimés sont présentées au tableau 3. Pour NT, les héritabilités réalisées sont faibles et non significatives en 1 re portée et deviennent significatives à partir de la $2^{\mathrm{e}}$ portée. Cette tendance ne se retrouve pas pour les héritabilités mère-fille, qui sont uniformément faibles et non significatives. L'analyse de NV donne des résultats similaires.

\section{TABleau 2}

Résultats de l'analyse univariate (modèle 3).

Results of univariate analysis (model 3).

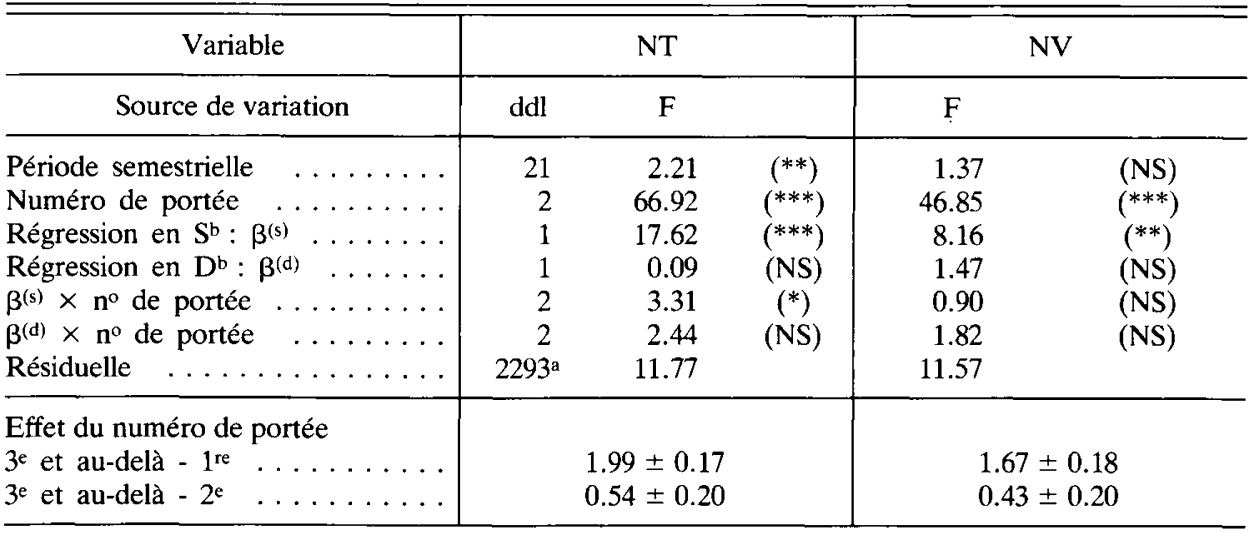

NS : non significatif.

: significatif $P<5$ p. 100

** : significatif $\mathrm{P}<1$ p. 100 .

significatif $P<1$ p. 1000.

2246 pour NV.

: S défini par l'équation (2b), D défini par l'équation (1). 


\section{TABLEAU 3}

Héritabilités estimées par le modèle (3).

Heritabilities estimated by model (3).

\begin{tabular}{|c|c|c|}
\hline Variable & NT & NV \\
\hline \multicolumn{3}{|c|}{ Héritabilité réalisée $\left(h_{r}^{2}\right)$} \\
\hline Toutes portées $\ldots \ldots$ & $\begin{array}{c}0.14 \pm 0.05 \\
(* * *)\end{array}$ & $\begin{array}{c}0.10 \pm 0.05 \\
\left(^{*}\right)\end{array}$ \\
\hline $1^{\mathrm{rc}}$. & $0.03 \pm 0.06$ & $0.04 \pm 0.06$ \\
\hline $2^{\mathrm{c}} \quad \ldots \ldots$ & $\begin{array}{c}0.26 \pm 0.08 \\
(* *)\end{array}$ & $0.14 \pm 0.08$ \\
\hline $3^{\mathrm{c}}$ et plus & $\begin{array}{c}0.18 \pm 0.07 \\
(* *)\end{array}$ & $0.14 \pm 0.07$ \\
\hline
\end{tabular}

Héritabilité mère-fille $\left(\mathrm{h}^{2}\right)$

\begin{tabular}{l|r|r}
\hline Toutes portées $\ldots \ldots \ldots \ldots \ldots \ldots \ldots$ & $0.02 \pm 0.03$ & $0.05 \pm 0.03$ \\
$1^{\text {re }} \ldots \ldots \ldots \ldots \ldots \ldots \ldots \ldots \ldots$ & $0.06 \pm 0.05$ & $0.05 \pm 0.05$ \\
$2^{\mathrm{e}} \quad \ldots \ldots \ldots \ldots \ldots \ldots \ldots \ldots \ldots \ldots$ & $-0.10 \pm 0.06$ & $0.05 \pm 0.06$ \\
$3^{\mathrm{e}}$ et plus $\ldots \ldots \ldots \ldots \ldots \ldots \ldots$ & $0.07 \pm 0.05$ & $0.11 \pm 0.05$ \\
& & $\left(^{*}\right)$ \\
\hline \hline
\end{tabular}

TABLEau 4

Différentielles de sélection des pères (*) (moyennes pondérées par le nombre de portées). Average sire selection differentials (*) (weighted by the number of daughter litters).

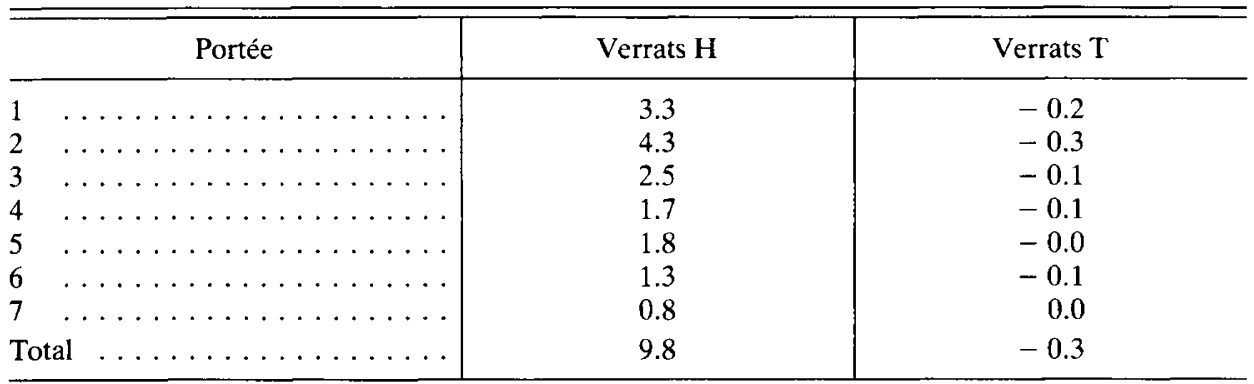

(*) Calculées en fonction des différentielles de sélection d (par portée) et $\mathrm{D}$ (global) appliquées aux mères de verrats. (*) Calculated from the boar dam selection differentials $d$ and $D$. 


\section{B. Approche multivariate}

La sélection effectuée sur les mères des verrats $H$ et $T$ est présentée au tableau 4 pour les 7 portées prises en compte. Il apparaît clairement que la sélection est nettement plus intense sur les 2 premières portées que sur les suivantes dans la lignée $\mathrm{H}$. Par ailleurs, une contre-sélection sur la prolificité, faible mais assez systématique, existe dans le choix des verrats de la lignée $T$.

Le modèle multivariate (5) a été appliqué pour $\mathrm{n}=7$ après calcul du vecteur $\mathbf{S}$ propre à chaque père, sur la base des variances résiduelles du modèle (3) et d'un coefficient de corrélation de 0,15 entre les portées d'une même truie. Par ailleurs, compte tenu des résultats du modèle (3), l'effet de la prolificité maternelle $\left(\beta^{(d)}\right)$ n'a pas été inclus dans le modèle (5).

Les résultats de l'analyse sont présentés au tableau 5. Les covariances génétiques obtenues sont estimées avec une faible précision et non significatives, à l'exception de celle qui lie la $3^{e}$ portée des mères de verrat et les portées de rang égal ou supérieur à 3 des filles. Bien que les estimées soient peu fiables, et que des valeurs anormalement élevées soient obtenues notamment à partir de la $3^{\mathrm{e}}$ portée, la tendance générale indique des covariances génétiques plus faibles quand il s'agit des $1^{\text {res }}$ portées des filles (1 $1^{\text {re }}$ ligne du tableau 5).

\section{Discussion et conclusions}

\section{A. Le modèle et ses approximations}

Dans l'analyse qui vient d'être présentée, les variations dues à l'élevage n'ont pas été prises en compte, à cause de la faible taille moyenne des troupeaux concernés. De plus, alors que toutes les $1^{\text {res }}$ portées des filles mises en élevage sont obtenues par insémination artificielle (cf. II.A.), une part importante des portées suivantes résulte de monte naturelle. Mais ce facteur, qui n'était pas répertorié, ne pouvait pas être inclus dans l'analyse. Il s'agit là de 2 causes de variation dont l'importance est bien établie (voir, par exemple, BRANDT \& GLODEK, 1984). Le fait de les ignorer réduit automatiquement la précision des comparaisons effectuées, comme en témoignent les variances résiduelles du tableau 2, assez nettement supérieures aux valeurs couramment admises intra-troupeau. L'incidence sur les paramètres génétiques est difficile à évaluer, mais il n'y a pas de raison de suspecter a priori des confusions statistiques susceptibles de les fausser. Par contre, le pourcentage plus faible d'insémination artificielle chez les multipares peut contribuer à expliquer la différence de prolificité entre multipares et primipares du tableau 2, qui est supérieure aux valeurs habituellement trouvées.

\section{B. Effets maternels}

Bien que la régression mère-fille soit estimée ici sur le phénotype moyen de la mère de la truie, et non pas sur la taille de la portée dont celle-ci est issue, les faibles valeurs d'héritabilité obtenues suggèrent l'existence d'effets maternels défavorables aux truies nées dans des portées importantes. En supposant que la portée moyenne de sa mère est celle dans laquelle la truie est née, le coefficient $\mathrm{m}$, défini par FALCONER (cité 


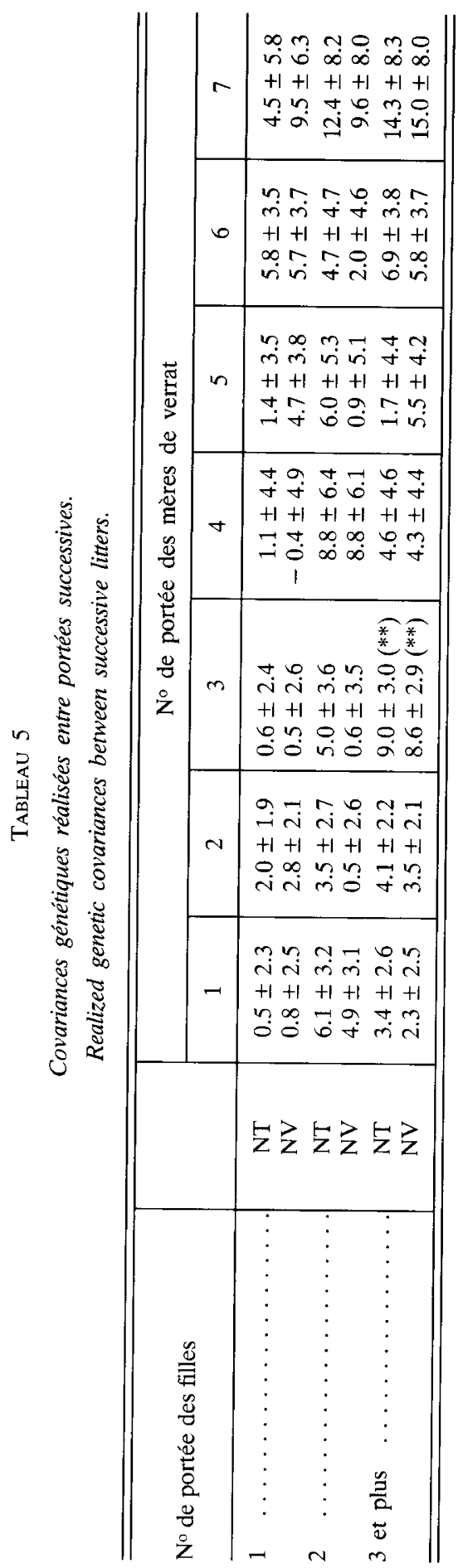


par VAN DER STEEN, 1985b) pour exprimer ces effets, est ici égal à $-0,06$ pour NT et $-0,02$ pour NV. Ces estimations sont assez nettement inférieures à la valeur moyenne de - 0,125 calculée par VAN DER STEEN (1985a) d'après les résultats de la littérature sur souris et porc.

Par ailleurs, l'évolution du phénomène au cours des portées successives (valeurs de m pour NT égales à $0,02,-0,17,-0,01$ dans l'ordre des numéros de portée d'après les estimations du tableau 3) n'indique pas de tendance régulière. Elle ne permet donc pas de trancher entre l'hypothèse de RoBIson (1979) qui prévoit une atténuation des effets maternels défavorables avec l'âge de la truie (à cause du retard de croissance des jeunes femelles élevées dans de grandes portées), et celle de RUTLEDGE (1980) qui prédit une persistance de ces effets durant toute la carrière de la truie (à cause d'un effet précoce sur la taille du «pool» de follicules primordiaux).

\section{Effets génétiques}

Les estimations d'héritabilités réalisées supposent en toute rigueur l'absence d'effet sélectif «parasite " pouvant affecter des caractères liés à la variable sélectionnée. Or, comme cela a été indiqué plus haut (en II.A.), les 2 lignées $\mathrm{H}$ et $\mathrm{T}$ sont soumises à une sélection sur croissance et adiposité. Cette sélection est plus intense dans la lignée $\mathrm{T}$ que dans la lignée $\mathrm{H}$ et la différence est accentuée par l'augmentation de l'intervalle de génération mère-fils de la lignée $H(3,7$ ans pour $H$ contre 1,6 pour $T)$ qui induit un retard génétique de la lignée $H$ pour ces caractères. Des comparaisons effectuées sur les candidats à la sélection des 2 sexes le confirment, notamment pour la vitesse de croissance (résultats non publiés). Dans cette situation, une corrélation génétique positive (par exemple) entre croissance et prolificité aurait pour effet de diminuer la différence de prolificité entre $\mathrm{H}$ et $\mathrm{T}$ et conduirait à sous-estimer l'héritabilité réalisée. Mais les liaisons génétiques mises en évidence entre la prolificité et les caractères de production sont généralement peu marquées (STEANE, 1981 ; Bolet et al., 1984). Leur incidence sur nos estimations devrait donc être minime. Il faut remarquer par ailleurs que dans la mesure où les comparaisons du tableau 4 refléteraient une corrélation génétique négative entre l'indice croissance-adiposité et la prolificité (ce qui n'est cependant pas prouvé), la prise en compte de différentielles de sélection négatives dans la lignée $\mathrm{T}$ tend à corriger les effets de cette liaison.

Il faut mentionner aussi les difficultés de calcul aussi bien que d'interprétation de l'héritabilité réalisée quand il y a recouvrement des générations successives, par suite des différences génétiques entre les cohortes parentales engendrées par la sélection elle-même, comme cela a été discuté récemment par JAMES (1986). Dans l'expérience analysée ici, l'hypothèse est faite que la population des femelles d'où sont tirées les " hyperprolifiques » n'est pas elle-même sélectionnée sur la prolificité et qu'il n'y a donc pas de différence génétique entre les cohortes parentales.

Globalement, les espérances théoriques fondées sur la lignée mâle « hyperprolifique " de Rouillé (LEGAult \& GRUAND, 1976) semblent se réaliser en élevage, avec un gain de 0,8 porcelet né et 0,6 porcelet né vivant chez les filles des verrats $H$, en supposant le critère de sélection $\mathrm{D}$ des truies hyperprolifiques égal à 12 en moyenne. Des gains tout à fait similaires ont été obtenus en race pure et en croisement dans une expérience anglaise (voir les résultats de BICHARD rapportés par LEGAULT, 1985, p. 161). Toutefois, les analyses présentées ici mettent en évidence une hétérogénéité des paramètres génétiques réalisés selon le numéro de portée, l'héritabilité réalisée de NT et NV en $1^{\text {re }}$ portée semblant plus faible que dans les portées ultérieures. Ces 
résultats sont à rapprocher des observations faites en troupeaux expérimentaux (LEGAULT et al., 1981 ; Bolet et Legault, 1982) qui révélaient un gain de prolificité chez les filles de verrats de la lignée $H$ multipares mais non chez les primipares.

Notre estimation des paramètres génétiques réalisés constitue une approche génétique de cette différence entre $11^{\text {re }}$ portée et portée d'ordre supérieur, jusqu'ici interprétée d'un point de vue physiologique (Bolet, 1984 ; Bolet et al., 1986). L'examen des données permet d'écarter l'existence d'une sélection des filles de verrats $\mathrm{H}$ sur leur prolificité en $1^{\text {re }}$ portée. Nos résultats nous conduisent donc à remettre en cause l'hypothèse selon laquelle les portées successives d'une truie sont l'expression d'un même caractère génétique. Nous ne disposions pas ici d'un dispositif expérimental permettant d'estimer précisément les corrélations génétiques entre performances successives d'une truie. Toutefois, les valeurs obtenues par divers auteurs, bien que très variables, présentent une tendance vers des corrélations génétiques inférieures à 1 , et généralement plus faibles entre la $1^{\text {re }}$ portée et les portées suivantes (HALEY et al., 1986). Ainsi, TARTAR (1981) démontre que, en sélectionnant sur la moyenne des 5 premières portées, la réponse attendue en $3^{\mathrm{e}}$ portée est le double de celle attendue en $1^{\text {re }}$ portée, lorsque des corrélations génétiques inférieures à 1 entre portées de rangs différents sont prises en compte. Dans le cas présent, cet effet pourrait expliquer la réponse élevée obtenue en $2^{e}$ portée, effet qui est renforcé par la pression de sélection maximale qui lui est appliquée (tableau 4). Cependant, pour expliquer la faible réponse observée en $1^{\text {re }}$ portée il faut supposer une héritabilité véritablement plus faible du caractère " $1^{\text {re }}$ portée ".

En conclusion, se pose le problème des limites de ce type de schéma de sélection (Avalos \& Smith, 1987). En effet, si les femelles de la population de base sont inséminées systématiquement avec les verrats de la lignée hyperprolifique, le niveau génétique de la population va lui-même s'améliorer au cours du temps. Aussi, tant qu'il sera possible de détecter des animaux exceptionnels, l'évolution continue du niveau génétique de la population pourra théoriquement se poursuivre.

Reçu le 21 novembre 1986.

Accepté le 26 février 1987.

\section{Références bibliographiques}

Avalos E., Sмith C., 1987. Genetic improvement of litter size in pigs. Anim. Prod., 44, 153-164.

Bichard M., David P.J., 1985. Effectiveness of genetic selection for prolificacy in pigs. J. Reprod. Fert., Suppl. 33, 127-138.

BOlET G., 1984. Timing and extent of embryonic mortality in pigs, sheep and goats. Genetic variability. "Embryonic mortality in farm animals ", E.E.C. Seminar, Brussels, December 11-12, 1984, Sreenan J.M., Diskin M.G. (ed.), 12-43, Martinus Nijhoff publishers, Brussels.

Bolet G., Legault C., 1982. New aspects of genetic improvement of prolificacy in the pig. 2nd World Congress on Genetics Applied to Livestock Production, Madrid, October 4-8, 1982, 5. 548-567, Editorial Garsi, Madrid.

Bolet G., Tartar M., Laloé D., Felgines C., 1984. Efficacité théorique de la sélection sur la prolificité chez le porc. Possibilité de modifier l'indice actuel. 16 Journées de la Recherche porcine en France, Paris, 1-3 février, 1984, 475-480, ITP, Paris (Ann. Zootech., 33, 419 (abst.)).

Bolet G., Martinat-Botté F., Locatelli P., Gruand J., Terqui M., Berthelot F., 1986. Components of prolificacy of hyperprolific Large-White sows compared with the Meishan and LargeWhite breeds. Génét. Sél. Evol., 18, 333-342. 
Brandt H., Glodek P., 1984. Der Einfluss der künstlichen Besamung auf die Wurfgrösse beim Schwein. Züchtungskunde, 56, 27-35.

Foulley J.L., 1981. La régression parent-descendant dans le cas d'un nombre variable de mesures par parent. Ann. Génét. Sél. Anim., 13, 181-188.

Haley C.S., Avalos E., Smith C., 1986. A review of selection for reproductive performance in the pig. 37th Annual Meeting of the European Association for Animal Production, Budapest, September 1-4, 1986, Summaries, 1, 102-103, EAAP, Rome.

JAMES J.W., 1986. Cumulative selection differentials and realized heritabilities with overlapping generations. Anim. Prod., 42, 411-415.

Legault C., 1985. Selection of breeds, strains and individual pigs for prolificacy. J. Reprod. Fert., Suppl. 33, 151-166.

Legault C., Gruand J., 1976. Amélioration de la prolificité des truies par la création d'une lignée « hyperprolifique " et l'usage de l'insémination artificielle : principe et résultats expérimentaux préliminaires. 8es Journées de la Recherche porcine en France, Paris, 4-6 février, 1976, 201-206, ITP, Paris.

Legault C., Gruand J., Bolet G., 1981. Résultats de l'utilisation en race pure et en croisement de la lignée dite "hyperprolifique ". 13es Journées de la Recherche porcine en France, Paris, 4-5 février, 1981, 255-260, ITP, Paris.

Noguera J.L., Felgines C., Legault C., 1983. Evolution de 1972 à 1981 des composantes de la productivité numérique des truies dans 325 troupeaux français. Variations régionales et saison-

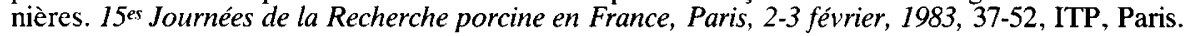

Ollivier L., 1974. La régression parent-descendant dans le cas de descendances subdivisées en familles de taille inégale. Biometrics, 30, 59-66.

Ollivier L., 1985. Dix ans d'une expérience de sélection sur des verrats utilisés en insémination artificielle. III. Paramètres génétiques réalisés. Génét. Sél. Evol., 17, 481-498.

Ollivier L., Bolet G., 1981. La sélection sur la prolificité chez le porc : résultats d'une expérience de sélection sur dix générations. 13 $3^{\text {es }}$ Journées de la Recherche porcine en France, Paris, 4-5 février, 1981, 261-268, ITP, Paris.

Ollivier L., Runavot J.P., Dagorn J., Gueblez R., Jehanno J., Keristr R., Legault C., Molenat M., Sellier P., 1986. La loi sur l'élevage de 1966: un bilan de 20 années de sélection rationnelle du porc en France. 18es Journées de la Recherche porcine en France, Paris, 29-31 janvier, 1986, 203-236, ITP, Paris.

Robison O.W., 1979. The influence of maternal effects on the efficiency of selection. 30th Annual Meeting of the European Association for Animal Production, Harrogate, July 22-26, 1979.

Rutledge J.J., 1980. Fraternity size and swine reproduction. 1. Effect on fecundity of gilts. 2. Genetical consequences. J. Anim. Sci., 51, 868-874.

Steane D., 1981. Antagonistic traits in pig breeding. Livest. Prod. Sci., 8, 407-418.

TARTAR M., 1981. Analyse critique des possibilités d'estimation du potentiel génétique des truies reproductrices. 65 p., D.E.A. de Génétique quantitative et appliquée, Université Paris-Sud.

VAN DER STEen H.A.M., 1985a. Maternal influence mediated by litter size during the suckling period on reproduction traits in pigs. Livest. Prod. Sci., 13, 147-158.

VAN DER STEEN H.A.M., 1985b. The implication of maternal effects for genetic improvement of litter size in pigs. Livest. Prod. Sci., 13, 159-168. 